\title{
Effects of Daminozide, Paclobutrazol, and Uniconazole Treatments on 'McIntosh' Apples at Harvest and Following Storage
}

\author{
D.C. Elfving' \\ Horticultural Research Institute of Ontario, Box 587, Simcoe, Ont. N3Y 4N5, Canada \\ E.C. Lougheed ${ }^{2}$ \\ Department of Horticultural Science, University of Guelph, Guelph, Ont. N1G 2W1, Canada
}

C.L. Chu' and R.A. Cline

Horticultural Research Institute of Ontario, Vineland Station, Ont. LOR 2E0, Canada

Additional index words. Malus domestica, Alar, Cultar, Prunit, stion, strain

\begin{abstract}
Foliar daminozide (DZ) applications to 'McIntosh' apple trees (Malus domestics Borkh.) increased fruit color, reduced preharvest drop, resulted in greater firmness at harvest and after air storage, delayed starch hydrolysis, and reduced fruit ethylene production at harvest and after storage. Foliar paclobutrazol (PBZ) reduced preharvest drop and flesh firmness loss if applied within 5 weeks after full bloom (WAFB). Later applications had no effect. PBZ did not influence the progress of starch hydrolysis or ethylene production at harvest but reduced poststorage ethylene production in one season. Stem-cavity browning and brown core were increased by PBZ applied at 5 and 9 WAFB in 1987. In 1988, fruit soluble solids content (SSC) was reduced by a double application of PBZ and by uniconazole (UCZ). UCZ had little effect on 'McIntosh' fruit other than the reduction in SSC. PBZ applications were less consistent in their effects than DZ. Chemical names used: butanedioic acid mono(2,2-dimethylhydrazide) (daminozide); B-[(4-chlorophenyl) methyl] $\alpha$ - -(1,l-dimethylethyl)-1H-1,2,4-triazole-1-ethanol (paclobutrazol); B-[(4-chloro-

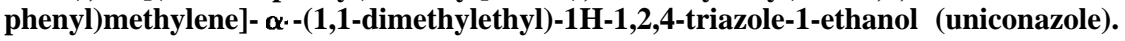

Preharvest drop, uneven fruit maturity, poor red color development at fruit maturity, excessive loss of fruit tissue firmness, and fruit quality deterioration during and after storage are problems typically associated with 'McIntosh', the predominant apple cultivar grown in eastern Canada. Daminozide (DZ, Uniroyal Chemical, Middlebury, Corm.) applied in midsummer has been widely used to accelerate color development, delay and synchronize fruit maturation, control preharvest drop, and maintain better fruit quality during storage (Edgerton and Blanpied, 1970; Forshey, 1970; Greene et. al., 1977; Looney, 1968a, 1968b, 1971, 1975a, 1975b; Miller and Lougheed, 1971; Williams et al., 1964). This material was removed from commercial sales channels in Canada and the United States by the manufacturer in June 1989.

Elfving et al. (1987) reported that paclobutrazol (PBZ, ICI Americas, Goldsboro, N. C.) applied to 'McIntosh' apple trees shortly after bloom affected fruit quality characteristics similar to DZ. Only foliar sprays were effective, and carryover effects on shoot or fruit growth the following season from either foliar or soil treatments were not accompanied by effects on fruit physiological behavior before or following air storage. In one season, Luo et al. $(1987,1989)$ reported some similar effects of PBZ foliar applications on 'Bramley's Seedling' and 'Golden Delicious' apples in the United Kingdom. The research described in this report was designed to evaluate the potential of PBZ to replace DZ on 'McIntosh' apple. Uniconazole (UCZ,

\footnotetext{
Received for publication 8 Nov. 1989. Mention of a product or trade name does not constitute a guarantee or warranty of the product by the Horticultural Research Inst. of Ontario nor an endorsement over similar products not mentioned. The cost of publishing this paper was defrayed in part by the payment of page charges. Under postal regulations, this paper therefore must be hereby marked advertisement solely to indicate this fact.

'Research Scientist.

${ }^{2}$ Professor.
}

Chevron Chemical, Richmond, Calif.) was included only in 1988.

\section{Materials and Methods}

Three field trials were carried out at the Horticultural Experiment Station, Simcoe, Ont., Canada, between 1986 and 1988, All trials used single, whole-tree plots in randomized completeblock designs. Each block contained six trees each of four stions ('Geneva McIntosh'/MM.106, 'Macspur McIntosh'/MM. 106, 'Morspur McIntosh'/MM.106, and 'Macspur McIntosh'/ MM.111), with the trees of each stion grouped together and the stion groups randomized within each block. The trees were planted in 1971 at a spacing of $5.5 \times 7.3 \mathrm{~m}$ on a well-drained Wilsonville soil composed mainly of gravelly, sandy till and were $=3.3 \mathrm{~m}$ high during the experiments.

All growth regulators were applied using a Rittenhouse selfpropelled air-blast sprayer (M.K. Rittenhouse and Sons, Jordan, Ont., Canada) equipped with a Hiniker computerized flow-control system (Hiniker, Mankato, Minn.) for precise control of spray volume. This method permitted dilute spray coverage with minimal runoff. A cloth panel, 4-m square, mounted on a truck and driven on the opposite side of the tree in tandem with the sprayer prevented cross-contamination of trees in adjacent rows during spray application.

Mid-shoot leaves from current-season shoot growth were collected from each test tree each August and processed for foliar analysis according to the methods of Bodnar et al. (1983). Postharvest and poststorage fruit characteristics were evaluated at the Horticultural Research Inst. of Ontario (HRIO) postharvest laboratory in Vineland (1986) and at the Dept. of Horticultural Science (DHS), Univ. of Guelph (1987 and 1988).

Daminozide and paclobutrazol, 1986 (Expt. 1). DZ (750 mg.liter $\left.{ }^{-1}\right)$ or PBZ $\left(125,250\right.$, or $500 \mathrm{mg} \cdot$ liter $\left.^{-1}\right)$ were applied dilute, as described, at 1900 liters.ha ${ }^{-1}$ on 1 July 1986 to one whole tree per stion in each of two blocks (eight replications). 
Controls remained unsprayed. Full bloom occurred on 8 May. Apple samples were harvested from each tree on 8 Sept. and again on 16 Sept. Postharvest evaluations were carried out at HRIO on a subsample of each replicate. Apples harvested on 8 Sept. were held in low-oxygen (LO) storage $\left(1.0 \% \mathrm{O}_{2}\right.$ and $1.0 \%$ $\mathrm{CO}_{2}$ ) and apples harvested on 16 Sept. were held in controlledatmosphere (CA) storage $\left(2.5 \% \mathrm{O}_{2}\right.$ and $\left.2.5 \% \mathrm{CO}_{2}\right)$. All apples were held at $1 \mathrm{C}$ and $90 \%$ to $95 \% \mathrm{RH}$ for 23 weeks before poststorage evaluation.

Daminozide and paclobutrazol, 1987 (Expt. 2). DZ (750 $\mathrm{mg} \cdot$ liter $^{-1}$ ) was applied 7 July and PBZ (500 mg.liter-1) plus $0.1 \%$ Tween-20 (v/v) (Atkemix, Brantford, Ont., Canada) was applied to separate sets of trees on 21 May, 11 June, or 7 July by air-blast sprayer. Full bloom occurred on 8 May. Each treatment was applied to one whole tree in each of four stions in each of two blocks (eight replications). Dilute spray volumes used were 1700 liters.ha ${ }^{-1}$ (21 May), 2000 liters.ha ${ }^{-1}$ (11 June), and 2250 liters. ha ${ }^{-1}(7 \mathrm{July})$. Controls remained unsprayed. Apple samples were harvested from each tree on 3 Sept. and again on 10 Sept. Postharvest evaluations were carried out at DHS on a subsample from each replicate. The remaining fruit was held in air at $0.5 \mathrm{C}$ and $90 \%$ to $95 \% \mathrm{RH}$ for 19 weeks before poststorage evaluations.

Daminozide, paclobutrazol, and uniconazole, 1988 (Expt. 3). DZ (750 mg.liter ${ }^{-1}$ ), PBZ [500 mg.liter ${ }^{-1}$ plus $0.1 \%$ Tween$20(\mathrm{v} / \mathrm{v})]$, or UCZ [250 mg.liter ${ }^{-1}$ plus $0.1 \%$ Tween-20 (v/v)] were applied dilute, as described, to one whole tree in each of four stions in each of two blocks (eight replications). DZ was applied at 2250 liters $\cdot$ ha $^{-1}$ on 28 June. PBZ and UCZ were applied once at 1700 liters.ha ${ }^{-1}$ on 31 May or applied to different trees on 31 May, as described, with a repeat application at 2000 liters $\cdot \mathrm{ha}^{-1}$ on 4 June. Controls remained unsprayed. Full bloom occurred on 17 May. Apple samples were harvested from each tree on 6 Sept. and again on 13 Sept. Postharvest and poststorage evaluations and storage term and conditions were the same as for Expt. 2.

Evaluation of fruit. In each trial, one limb containing a representative crop sample of not fewer than 40 fruits was chosen for estimation of preharvest fruit color development (Forshey and Elfving, 1979). Fruit color was visually compared with a color photograph of 'McIntosh' apple epidermis exhibiting the appropriate color intensity as measured by a Techwest model RD-3 apple color meter (Techwest Enterprises, Vancouver, B. C., Canada) (Agr. Canada, 1987). A fruit was judged to have acceptable color when $30 \%$ of its surface displayed the appropriate intensity of red ('Canada Fancy' grade) (Agr. Canada, 1987). Pedicel length was determined by longitudinally sectioning the fruit and pedicel from 25 fruit at harvest and measuring pedicel length with a Mitutoyo vernier caliper (Cole-Parmer, Chicago) from the abscission zone to the junction of the fruit epidermis. In 1989, pedicels were measured on 25 fruit/tree 4 weeks after bloom. Pedicels had completed their elongation by this time (Prive et al., 1988).

Fruit flesh firmness was evaluated with an Effegi (Alfonsine, Italy) penetrometer (11 1-mm tip) by making one measurement each on pared flesh on opposite sectors of each of 10 apples per replicate. Juice expressed, from the apples during the firmness measurements was collected, and soluble solids concentration (SSC) was assessed on the composite juice sample with an Atago model N1 hand refractometer (American Optical, Buffalo, N.Y.). Fruit maturation was assessed on the same 10 apples by use of the starch-iodine test and rating starch patterns against a 'McIntosh' standard (Phillips and Poapst, 1952; Poapst et al., 1959; Priest and Lougheed, 1981; Smith et al., 1979). Following storage, 10 apples per replicate were assessed for tissue firmness and SSC. Disorders were rated visually on 20 fruit (HRIO) or 25 fruit (DHS) per replicate after 1 week at room temperature $(\approx 20 \mathrm{C}$ ) on a scale from 0 (no symptoms) to 3 (severe symptoms).

Fruit ethylene levels were measured after storage in Expt. 1 on three apples per replicate using a Hewlett-Packard (Mississauga, Ont., Canada) 5880A gas chromatography equipped with a dual flame ionization detector. At harvest and following storage in 1987 and 1988, 10 apples per replicate were placed in a flow-through system (Lougheed and Franklin, 1968). The effluent air was automatically sampled and 3-ml samples were injected into a Beckman GC-5 gas chromatography (Beckman Instruments, Fullerton, Calif.) with a dual flame ionization detector (Lougheed et al., 1969) and thermal conductivity detectors. The latter detectors were used to measure $\mathrm{CO}_{2}$ levels. Rates of ethylene production and respiration, the latter as $\mathrm{CO}_{2}$ production, were calculated from levels of these gases in the effluent airstream.

Growth regulator treatments were randomized within each stion group in a factorial split-plot arrangement, with stion as the main plot and growth regulator as the sub-plot. All percentage data were transformed using the arcsin of the square root before analysis. Analyses of variance, single degree-offreedom contrasts, and Duncan's multiple range test were carried out using the General Linear Models procedure of the Statistical Analysis System program package (SAS Institute, Cary, N.C.).

\section{Results}

Experiment 1. Both DZ and PBZ increased the percentage of fruit with acceptable red color at harvest, but only DZ reduced preharvest drop (Table 1). Seed content and pedicel length in 1986 were not influenced by growth regulator treatments. Reduced pedicel length the following year indicated some carryover effects for DZ and an increased carryover effect with the higher PBZ concentration applied in 1986. No stion effects were observed for any characteristics.

Flesh of DZ-treated fruit was firmer at harvest and after 23 weeks of CA storage than the control (Table 2). DZ also retarded starch hydrolysis. Other at-harvest and post CA-storage characteristics were unaffected by any growth regulator treatment. Results from apples stored under LO conditions were similar (data not shown). Some stion differences were observed in fruit size, tissue firmness, SSC, and starch index, with differences after storage reflecting fruit condition at harvest.

Experiment 2. The percentage of fruit with acceptable red color at harvest in 1987 was high and showed no effect of any treatment (Table 3). Preharvest drop was reduced only by DZ. Seed content was lower only in the earliest PBZ treatment, applied $\approx 2 \mathrm{WAFB}$. Pedicel length was unaffected the year of treatment, but in the following year, possible PBZ carryover was indicated for the last of the three treatments, applied $\approx 9$ weeks AFB. Stions showed no differences in any characteristic.

Fruit weight at harvest was unaffected by any growth regulator treatment (Table 4). Flesh firmness was greater for fruit treated with DZ or PBZ 2 WAFB, but the starch index and ethylene evolution showed significant retardation of maturation only for DZ-treated fruit. Fruit weight at harvest was lowest for 'Morspur McIntosh'/MM. 106, even though trunk cross-sec- 
Table 1. Effects of daminozide (DZ) and paclobutrazol (PBZ) foliar applications in 1986 on 'McIntosh' fruit at harvest in 1986 and 1987 (Expt. 1).

\begin{tabular}{|c|c|c|c|c|c|}
\hline \multirow[b]{2}{*}{ Factor } & \multicolumn{4}{|c|}{1986} & \multirow{2}{*}{$\begin{array}{c}1987 \\
\text { Pedicel } \\
\text { length } \\
(\mathrm{mm})\end{array}$} \\
\hline & $\begin{array}{c}\text { Acceptable } \\
\text { red color } \\
\text { (\% Canada Fancy) }\end{array}$ & $\begin{array}{c}\text { Preharvest } \\
\text { drop } \\
(\% \text { of yield })\end{array}$ & $\begin{array}{l}\text { Seeds } \\
\text { per } \\
\text { fruit }\end{array}$ & $\begin{array}{l}\text { Pedicel } \\
\text { length } \\
(\mathrm{mm})\end{array}$ & \\
\hline \multicolumn{6}{|l|}{ Chemical (mg.liter $\left.{ }^{-1}\right)^{2}$} \\
\hline DZ 750 & 63 & 31 & 7.8 & 13.7 & 12.8 \\
\hline Control & 44 & 67 & 7.4 & 14.2 & 14.1 \\
\hline PBZ 125 & 62 & 73 & 7.8 & 13.8 & 12.5 \\
\hline PBZ 250 & 50 & 67 & 7.4 & 13.4 & 11.9 \\
\hline PBZ 500 & 67 & 64 & 7.2 & 14.3 & 11.5 \\
\hline \multicolumn{6}{|l|}{ Significance $^{y}$} \\
\hline DZ vs. control & $* *$ & $* * * *$ & NS & NS & $* *$ \\
\hline PBZ linear & $*$ & NS & NS & NS & $* *$ \\
\hline PBZ quadratic & NS & NS . & NS & NS & NS \\
\hline PBZ cubic & $* *$ & NS & NS & NS & NS \\
\hline \multicolumn{6}{|l|}{ Stion $^{x}$} \\
\hline Geneva/MM.106 & $50 \mathrm{a}$ & $63 \mathrm{a}$ & $7.1 \mathrm{a}$ & $14.3 \mathrm{a}$ & $12.5 \mathrm{a}$ \\
\hline Macspur/MM.106 & $56 a$ & $56 a$ & $7.1 \mathrm{a}$ & $14.2 \mathrm{a}$ & $12.5 \mathrm{a}$ \\
\hline Morspur/MM.106 & $60 \mathrm{a}$ & $64 \mathrm{a}$ & $7.7 \mathrm{a}$ & $13.8 \mathrm{a}$ & $13.0 \mathrm{a}$ \\
\hline Macspur/MM.111 & $63 \mathrm{a}$ & 59 a & $8.0 \mathrm{a}$ & $13.2 \mathrm{a}$ & $12.0 \mathrm{a}$ \\
\hline
\end{tabular}

${ }^{2}$ Applications with an air-blast sprayer at 1900 liters $\cdot$ ha $^{-1}$ (1 July). Full bloom 8 May. ${ }^{y}$ Analysis by single degree-of-freedom contrasts. Regression contrasts include control. Significant at $P=0.05(*), 0.01(* *)$, or $0.0001(* * * *)$. Each mean contains 8 observations. ${ }^{x}$ Mean separation within columns by Duncan's multiple range test $(P=0.05)$. No chemical $x$ stion interactions. Each mean contains 10 observations.

Table 2. Effects of daminozide (DZ) and paclobutrazol (PBZ) foliar applications in 1986 on 'McIntosh' fruit quality characteristics at harvest in 1986 and following 23 weeks in CA storage at 1C (Expt. 1).

\begin{tabular}{|c|c|c|c|c|c|c|c|c|c|}
\hline \multirow[b]{4}{*}{ Factor } & \multirow{2}{*}{\multicolumn{4}{|c|}{ Harvest }} & \multicolumn{5}{|c|}{ Poststorage } \\
\hline & & & & & \multirow{2}{*}{\multicolumn{2}{|c|}{$\begin{array}{l}\text { Flesh } \\
\text { firmness } \\
(\mathrm{N})\end{array}$}} & \multirow{3}{*}{$\begin{array}{l}\text { Soluble } \\
\text { solids } \\
\text { concn } \\
(\%)^{y}\end{array}$} & \multirow{3}{*}{$\begin{array}{c}\text { Core } \\
\mathrm{C}_{2} \mathrm{H}_{4} \\
\left(\mu \mathrm{l} \cdot \text { liter }^{-1}\right)^{\mathrm{y}}\end{array}$} & \multirow{3}{*}{$\begin{array}{c}\text { Brown } \\
\text { core } \\
(\%)^{y, x}\end{array}$} \\
\hline & \multirow{2}{*}{$\begin{array}{c}\text { Fruit } \\
\text { wt } \\
(\mathrm{g}) \\
\end{array}$} & \multirow{2}{*}{$\begin{array}{c}\text { Flesh } \\
\text { firmness } \\
(\mathrm{N})\end{array}$} & \multirow{2}{*}{$\begin{array}{c}\text { Soluble } \\
\text { solids } \\
(\%) \\
\end{array}$} & \multirow{2}{*}{$\begin{array}{l}\text { Starch } \\
\text { index } \\
\end{array}$} & & & & & \\
\hline & & & & & 1 day & 1 week $^{y}$ & & & \\
\hline \multicolumn{10}{|l|}{$\overline{\left.\text { Chemical (mg } \cdot \text { liter }^{-1}\right)^{\mathrm{w}}}$} \\
\hline DZ 750 & 160 & 63.1 & 12.2 & 5.6 & 51.0 & 49.4 & 11.6 & 324 & 0 \\
\hline Control & 160 & 60.2 & 12.1 & 6.3 & 46.8 & 44.9 & 11.8 & 381 & 0 \\
\hline PBZ 125 & 160 & 60.3 & 12.2 & 6.4 & 47.6 & 45.2 & 11.9 & 360 & 1 \\
\hline PBZ 250 & 160 & 61.7 & 12.3 & 6.5 & 45.6 & 44.1 & 12.1 & 365 & 2 \\
\hline PBZ 500 & 160 & 61.5 & 12.3 & 6.2 & 44.0 & 42.6 & 11.8 & 345 & 1 \\
\hline \multicolumn{10}{|l|}{ Significance $^{v}$} \\
\hline DZ vs. control & NS & * & NS & $*$ & $*$ & $*$ & NS & NS & NS \\
\hline PBZ linear & NS & NS & NS & NS & NS & NS & NS & NS & NS \\
\hline PBZ quadratic & NS & NS & NS & NS & NS & NS & NS & NS & NS \\
\hline PBZ cubic & NS & NS & NS & NS & NS & NS & NS & NS & NS \\
\hline \multicolumn{10}{|l|}{ Stion ${ }^{4}$} \\
\hline Geneva/MM.106 & $160 \mathrm{ab}$ & $60.4 \mathrm{~b}$ & $12.0 \mathrm{~b}$ & $6.1 \mathrm{ab}$ & $44.8 \mathrm{~b}$ & $42.7 \mathrm{~b}$ & $11.7 \mathrm{a}$ & 328 a & $0 \mathrm{a}$ \\
\hline Macspur/MM.106 & $170 \mathrm{a}$ & $60.2 \mathrm{~b}$ & $12.1 \mathrm{~b}$ & $5.8 \mathrm{~b}$ & $44.5 \mathrm{~b}$ & $42.0 \mathrm{~b}$ & $11.6 \mathrm{a}$ & $429 a$ & $1 \mathrm{a}$ \\
\hline Morspur/MM.106 & $150 \mathrm{~b}$ & $64.9 \mathrm{a}$ & $12.4 \mathrm{a}$ & $6.3 \mathrm{ab}$ & $53.4 \mathrm{a}$ & $52.8 \mathrm{a}$ & $12.1 \mathrm{a}$ & $317 \mathrm{a}$ & $0 \mathrm{a}$ \\
\hline Macspur/MM.111 & $160 \mathrm{~b}$ & $59.9 \mathrm{~b}$ & $12.3 \mathrm{a}$ & $6.6 \mathrm{a}$ & $45.3 \mathrm{~b}$ & $43.4 \mathrm{~b}$ & $12.0 \mathrm{a}$ & $347 \mathrm{a}$ & $3 a$ \\
\hline \multicolumn{10}{|l|}{ Harvest date" } \\
\hline 8 Sept. & $150 \mathrm{~b}$ & $64.0 \mathrm{a}$ & $12.1 \mathrm{a}$ & $5.5 \mathrm{~b}$ & & & & & \\
\hline 16 Sept. & $170 \mathrm{a}$ & $58.7 \mathrm{~b}$ & $12.3 \mathrm{a}$ & $6.9 \mathrm{a}$ & & & & & \\
\hline
\end{tabular}

${ }^{2}$ Mean treatment rating on a scale from 1 to 9 (immature to overmature).

Devaluation after 1 week at room temperature.

${ }^{x}$ Percentage of 10 -fruit samples showing moderate (rating of 2) to severe (rating of 3) symptoms.

"Applications with an air-blast sprayer at 1900 liters $\cdot$ ha $^{-1}$ (1 July). Full bloom 8 May. Each harvest mean contains 16 observations, each poststorage mean eight.

${ }^{v}$ Analysis by single degree-of-freedom contrasts. Nonsignificant (NS) or significant at $P=0.05(*)$.

"Mean separation by Duncan's multiple range test $(P=0.05)$ for stion or F test $(P=0.05)$ for harvest date. No interactions among factors. Each harvest mean for stion contains 20 observations, and each poststorage mean for stion contains 10 observations. Apples harvested on 16 Sept. were used for poststorage evaluation. 
Table 3. Effects of daminozide (DZ) and paclobutrazol (PBZ) foliar applications in 1987 on 'McIntosh' fruit at harvest in 1987 and 1988 (Expt. 2).

\begin{tabular}{|c|c|c|c|c|c|c|}
\hline \multirow[b]{2}{*}{ Factor } & \multirow[b]{2}{*}{$\begin{array}{l}\text { Application } \\
\text { date }\end{array}$} & \multicolumn{4}{|c|}{1987} & \multirow{2}{*}{$\begin{array}{l}1988 \\
\text { Pedicel } \\
\text { length } \\
(\mathrm{mm})\end{array}$} \\
\hline & & $\begin{array}{c}\text { Acceptable } \\
\text { red color } \\
\text { (\% Canada Fancy) }\end{array}$ & $\begin{array}{c}\text { Preharvest } \\
\text { drop } \\
\text { (\% of yield) }\end{array}$ & $\begin{array}{c}\text { Seeds } \\
\text { per } \\
\text { fruit } \\
\end{array}$ & $\begin{array}{l}\text { Pediçel } \\
\text { length } \\
(\mathrm{mm})\end{array}$ & \\
\hline \multicolumn{7}{|c|}{ Chemical (mg $\cdot$ liter $\left.^{-1}\right)^{z, y}$} \\
\hline Control & $\cdots$ & $85 a$ & $20 \mathrm{a}$ & $7.7 \mathrm{a}$ & $12.9 \mathrm{a}$ & $12.8 \mathrm{a}$ \\
\hline DZ 750 & 7 July & 91 a & $10 \mathrm{~b}$ & $7.7 \mathrm{a}$ & $12.6 \mathrm{a}$ & $11.8 \mathrm{a}$ \\
\hline PBZ 500 & 21 May & $97 \mathrm{a}$ & $12 a b$ & $6.8 \mathrm{~b}$ & $12.3 \mathrm{a}$ & $12.8 \mathrm{a}$ \\
\hline PBZ 500 & 11 June & 92 a & $12 \mathrm{ab}$ & $8.0 \mathrm{a}$ & $13.0 \mathrm{a}$ & $11.3 \mathrm{a}$ \\
\hline PBZ 500 & 7 July & 94 a & $19 \mathrm{ab}$ & $7.5 \mathrm{ab}$ & $11.8 \mathrm{a}$ & $7.9 \mathrm{~b}$ \\
\hline \multicolumn{7}{|l|}{ Stion ${ }^{y}$} \\
\hline Geneva/MM.106 & & $87 \mathrm{a}$ & $17 \mathrm{a}$ & $7.4 \mathrm{a}$ & $12.5 \mathrm{a}$ & $12.8 \mathrm{a}$ \\
\hline Macspur/MM.106 & & $88 \mathrm{a}$ & $18 \mathrm{a}$ & $7.2 \mathrm{a}$ & $12.5 \mathrm{a}$ & $10.9 \mathrm{a}$ \\
\hline Morspur/MM.106 & & $98 a$ & $13 \mathrm{a}$ & $8.0 \mathrm{a}$ & 13.0 a & $11.4 \mathrm{a}$ \\
\hline Macspur/MM.111 & & $94 \mathrm{a}$ & $11 a$ & $7.5 \mathrm{a}$ & $12.0 \mathrm{a}$ & $10.3 \mathrm{a}$ \\
\hline
\end{tabular}

${ }^{2}$ Applications with an air-blast sprayer at 1700 (21 May), 2000 (11 June), or 2250 liters $\cdot$ ha $^{-1}$ (7 July). Full bloom 8 May.

${ }^{y}$ Mean separation within factors and columns by Duncan's multiple range test $(P=0.05)$. No chemical $\times$ stion interactions. Each mean for chemical contains eight observations, for stion 10 observations.

Table 4. Effects of daminozide (DZ) and paclobutrazol (PBZ) foliar applications in 1987 on 'McIntosh' fruit quality characteristics at harvest in 1987 (Expt. 2).

\begin{tabular}{|c|c|c|c|c|c|c|c|}
\hline \multirow[b]{2}{*}{ Factor } & \multirow{2}{*}{$\begin{array}{l}\text { Application } \\
\text { date } \\
\end{array}$} & \multirow{2}{*}{$\begin{array}{c}\text { Fruit } \\
\text { wt } \\
(\mathrm{g})\end{array}$} & \multirow{2}{*}{$\begin{array}{c}\text { Flesh } \\
\text { firmness } \\
(\mathrm{N})\end{array}$} & \multirow{2}{*}{$\begin{array}{c}\text { Soluble } \\
\text { solids } \\
\text { concn } \\
(\%)\end{array}$} & \multirow{2}{*}{$\begin{array}{l}\text { Starch } \\
\text { index }\end{array}$} & \multicolumn{2}{|c|}{$\begin{array}{c}\mathrm{C}_{2} \mathrm{H}_{4} \\
\left(\mu \mathrm{l} \cdot \mathrm{kg}^{-1} \cdot \mathrm{hr}^{-1}\right)\end{array}$} \\
\hline & & & & & & 1 day & 3 days \\
\hline \multicolumn{8}{|c|}{ Chemical $\left(\mathrm{mg} \cdot \operatorname{liter}^{-1}\right)^{\mathrm{y}, \mathrm{x}}$} \\
\hline Control & --- & $140 \mathrm{a}$ & $67.0 \mathrm{~b}$ & $12.6 \mathrm{a}$ & $4.4 \mathrm{ab}$ & $9.2 \mathrm{a}$ & $49.5 \mathrm{a}$ \\
\hline DZ 750 & 7 July & $150 \mathrm{a}$ & $68.6 \mathrm{a}$ & $12.5 \mathrm{a}$ & $4.0 \mathrm{c}$ & $1.0 \mathrm{~b}$ & $11.4 \mathrm{~b}$ \\
\hline PBZ 500 & 21 May & $150 \mathrm{a}$ & 68.7 a & $12.4 \mathrm{a}$ & $4.3 \mathrm{bc}$ & $3.2 \mathrm{ab}$ & $40.4 \mathrm{a}$ \\
\hline PBZ 500 & 11 June & $140 \mathrm{a}$ & $66.7 \mathrm{~b}$ & $12.4 \mathrm{a}$ & $4.6 \mathrm{ab}$ & $6.8 \mathrm{ab}$ & $43.5 \mathrm{a}$ \\
\hline PBZ 500 & 7 July & $140 \mathrm{a}$ & $66.0 \mathrm{~b}$ & $12.4 \mathrm{a}$ & $4.7 \mathrm{a}$ & $9.0 \mathrm{a}$ & $54.0 \mathrm{a}$ \\
\hline \multicolumn{8}{|l|}{ Stion ${ }^{\mathrm{x}}$} \\
\hline Geneva/MM.106 & & $150 \mathrm{a}$ & $67.9 \mathrm{a}$ & $12.5 \mathrm{a}$ & $4.1 \mathrm{~b}$ & $8.4 \mathrm{a}$ & $39.7 \mathrm{a}$ \\
\hline Macspur/MM.106 & & $150 \mathrm{a}$ & $66.8 \mathrm{a}$ & $12.4 \mathrm{a}$ & $4.6 \mathrm{a}$ & $6.0 \mathrm{a}$ & $47.4 \mathrm{a}$ \\
\hline Morspur/MM.106 & & $130 \mathrm{c}$ & $67.9 \mathrm{a}$ & $12.5 \mathrm{a}$ & $4.5 \mathrm{a}$ & $3.3 \mathrm{a}$ & 26.6 a \\
\hline Macspur/MM.111 & & $140 \mathrm{~b}$ & $67.1 \mathrm{a}$ & $12.4 \mathrm{a}$ & $4.3 \mathrm{ab}$ & $5.3 \mathrm{a}$ & $50.5 a$ \\
\hline \multicolumn{8}{|l|}{ Harvest date } \\
\hline 3 Sept. & & $140 \mathrm{~b}$ & $68.4 \mathrm{a}$ & $12.5 \mathrm{a}$ & $4.0 \mathrm{~b}$ & $2.7 \mathrm{~b}$ & $24.0 \mathrm{~b}$ \\
\hline 10 Sept. & & $150 \mathrm{a}$ & $66.4 \mathrm{~b}$ & $12.4 \mathrm{a}$ & $4.7 \mathrm{a}$ & $8.2 \mathrm{a}$ & $51.5 \mathrm{a}$ \\
\hline
\end{tabular}

${ }^{2}$ Mean treatment rating on a scale from 1 to 9 (immature to overmature).

${ }^{y}$ Applications with an air-blast sprayer at 1700 (21 May), 2000 (11 June), or 2250 liters·ha ${ }^{-1}$ (7 July). Full bloom 8 May.

${ }^{x}$ Mean separation within factors and columns by Duncan's multiple range test $(P=0.05)$ for chemical and stion and by $\mathrm{F}$ test $(P=0.05)$ for harvest date. No interactions among factors. Each mean for chemical contains 16 observations, for stion 20 observations, and for harvest date 40 observations.

tional area and yield per tree for this stion were not significantly different from the others (data not shown). No other stion differences were observed.

Following 19 weeks of air storage, there were no significant differences in flesh firmness after 1 day at room temperature, but after 1 week, fruit treated with DZ or with PBZ at 2 or 5 WAFB retained slightly greater firmness (Table 5). Ethylene evolution was lower for treated than for untreated fruit, but the lowest ethylene evolution was associated with the last PBZ application, which did not lead to any tissue firmness differences. This treatment also exhibited significantly higher incidence of both stem-cavity browning and brown core; the brown core incidence for the last PBZ application was significantly higher than for all other treatments. Differences among stions were again noted in flesh firmness and incidence of disorders. These poststorage differences among stions did not appear to be related to condition at harvest.

Experiment 3. In 1988, the incidence of acceptable red color at harvest was increased by DZ and the double application of PBZ, but was unaffected by UCZ (Table 6). Preharvest drop and seed content were not influenced by any treatment. Pedicel length in 1988 and 1989 gave no indication of carryover effects. Differences among stions in color and-pedicel length were observed for the first time in 1988.

At harvest, fruit weight was unaffected by the growth regulator treatments (Table 7). Flesh firmness was greater in fruit treated with DZ or the double application of PBZ. DZ delayed starch hydrolysis compared to the double application of either PBZ or UCZ and lowered ethylene evolution relative to either $\mathrm{UCZ}$ treatment. Interestingly, $\mathrm{SSC}$ was significantly reduced 
Table 5. Effects of daminozide (DZ) and paclobutrazol (PBZ) foliar applications in 1987 on 'McIntosh' fruit quality characteristics following 19 weeks in air at $0.5 \mathrm{C}$ (Expt. 2).

\begin{tabular}{|c|c|c|c|c|c|c|c|c|}
\hline \multirow[b]{2}{*}{ Factor } & \multirow{2}{*}{$\begin{array}{c}\text { Application } \\
\text { date }\end{array}$} & \multicolumn{2}{|c|}{$\begin{array}{c}\text { Flesh } \\
\text { firmness } \\
(\mathrm{N})\end{array}$} & \multirow{2}{*}{$\begin{array}{c}\text { Soluble } \\
\text { solids } \\
\text { concn } \\
(\%)\end{array}$} & \multirow{2}{*}{$\begin{array}{c}\mathrm{C}_{2} \mathrm{H}_{4} \\
\left(\mu \mathrm{l} \cdot \mathrm{kg}^{-1} \cdot \mathrm{hr}^{-1}\right) \\
\end{array}$} & \multirow{2}{*}{$\begin{array}{c}\text { Stem } \\
\text { cavity } \\
\text { browning } \\
(\%)^{z}\end{array}$} & \multirow{2}{*}{$\begin{array}{c}\text { Brown } \\
\text { core } \\
(\%)^{z}\end{array}$} & \multirow{2}{*}{$\begin{array}{c}\begin{array}{c}\text { Internal } \\
\text { breakdown } \\
(\%)^{\mathrm{z}}\end{array} \\
\end{array}$} \\
\hline & & 1 day & 1 week $^{2}$ & & & & & \\
\hline \multicolumn{9}{|c|}{ Chemical (mg-liter $\left.{ }^{-1}\right)^{y, x}$} \\
\hline Control & -- & $41.7 \mathrm{ab}$ & $39.6 \mathrm{ab}$ & $12.4 \mathrm{ab}$ & $165 \mathrm{a}$ & $47 \mathrm{~b}$ & $35 \mathrm{~b}$ & $1 \mathrm{a}$ \\
\hline DZ 750 & 7 July & $41.6 \mathrm{~b}$ & $40.5 \mathrm{a}$ & $12.4 \mathrm{a}$ & $147 \mathrm{~b}$ & $51 \mathrm{ab}$ & $39 \mathrm{~b}$ & $2 a$ \\
\hline PBZ 500 & 21 May & $42.4 \mathrm{ab}$ & $40.7 \mathrm{a}$ & $12.2 \mathrm{a}$ & $154 \mathrm{~b}$ & $54 \mathrm{ab}$ & $26 \mathrm{~b}$ & $1 \mathrm{a}$ \\
\hline PBZ 500 & 11 June & $42.5 \mathrm{a}$ & $40.4 \mathrm{a}$ & $12.1 \mathrm{a}$ & $149 \mathrm{~b}$ & $60 \mathrm{ab}$ & $39 \mathrm{~b}$ & $1 \mathrm{a}$ \\
\hline PBZ 500 & 7 July & $41.9 \mathrm{ab}$ & $39.7 \mathrm{~b}$ & $12.0 \mathrm{a}$ & $136 \mathrm{c}$ & $63 a$ & $77 a$ & $2 a$ \\
\hline \multicolumn{9}{|l|}{ Stion $^{x}$} \\
\hline Geneva/MM.106 & & $42.5 \mathrm{a}$ & $41.0 \mathrm{a}$ & $12.2 \mathrm{a}$ & $151 \mathrm{a}$ & $52 \mathrm{~b}$ & $46 \mathrm{ab}$ & $2 a$ \\
\hline Màcspur/MM.106 & & $41.2 \mathrm{~b}$ & $39.8 \mathrm{~b}$ & $12.2 \mathrm{a}$ & 149 a & 65 a & 58 a & $3 a$ \\
\hline Morspur/MM.106 & & $42.9 \mathrm{a}$ & $41.0 \mathrm{a}$ & $12.2 \mathrm{a}$ & $157 \mathrm{a}$ & $50 \mathrm{~b}$ & $28 \mathrm{c}$ & $0 \mathrm{~b}$ \\
\hline Macspur/MM.111 & & $41.4 \mathrm{~b}$ & $38.9 \mathrm{c}$ & $12.3 \mathrm{a}$ & $144 \mathrm{a}$ & $53 \mathrm{~b}$ & $41 \mathrm{bc}$ & $1 a b$ \\
\hline \multicolumn{9}{|l|}{ Harvest date } \\
\hline 3 Sept. & & $42.9 \mathrm{a}$ & $40.5 \mathrm{a}$ & $12.1 \mathrm{~b}$ & $137 \mathrm{~b}$ & 55 a & $36 \mathrm{~b}$ & $0 \mathrm{~b}$ \\
\hline 10 Sept. & & $41.1 \mathrm{~b}$ & $39.9 \mathrm{~b}$ & $12.3 \mathrm{a}$ & $163 \mathrm{a}$ & $55 \mathrm{a}$ & $51 \mathrm{a}$ & $2 a$ \\
\hline
\end{tabular}

${ }^{2}$ Evaluation after 1 week at room temperature. Percentages are of 25 -fruit samples showing moderate (rating of 2) to severe (rating of 3) symptoms.

${ }^{y}$ Applications with an air-blast sprayer at 1700 (21 May), 2000 (11 June), or 2250 liters·ha' (7 July). Full bloom 8 May.

'Mean separation within factors and columns by Duncan's multiple range test $(P=0.05)$ for chemical and stion and by $\mathrm{F}$ test ( $P$ $=0.05$ ) for harvest date. No interactions among factors. Each mean for chemical contains 16 observations, for stion 20 observations, and for harvest date 40 observations.

Table 6. Effects of daminozide (DZ), paclobutrazol (PBZ), and uniconazole (UCZ) foliar applications in 1988 on 'McIntosh' fruit at harvest in 1988 and 4 weeks after bloom in 1989 (Expt. 3).

\begin{tabular}{|c|c|c|c|c|c|c|c|}
\hline \multirow{2}{*}{\multicolumn{2}{|c|}{ Factor }} & \multirow[b]{2}{*}{$\begin{array}{l}\text { Application } \\
\text { date(s) }\end{array}$} & \multicolumn{4}{|c|}{1988} & \multirow{2}{*}{$\begin{array}{c}1989 \\
\begin{array}{c}\text { Pedicel } \\
\text { length } \\
(\mathrm{mm})\end{array}\end{array}$} \\
\hline & & & $\begin{array}{c}\text { Acceptable } \\
\text { red color } \\
\text { (\% Canada Fancy) }\end{array}$ & $\begin{array}{l}\text { Preharvest } \\
\text { drop } \\
\text { (\% of yield) }\end{array}$ & $\begin{array}{c}\text { Seeds } \\
\text { per } \\
\text { fruit }\end{array}$ & $\begin{array}{l}\text { Pedicel } \\
\text { length } \\
(\mathrm{mm})\end{array}$ & \\
\hline \multicolumn{8}{|c|}{$\overline{\text { Chemical }\left(\mathrm{mg} \cdot \text { liter }^{-1}\right)^{z, y}}$} \\
\hline Control & & --- & $47 \mathrm{c}$ & $23 \mathrm{a}$ & $5.5 \mathrm{a}$ & $13.1 \mathrm{a}$ & $18.0 \mathrm{a}$ \\
\hline DZ 750 & & 28 June & $65 \mathrm{a}$ & $13 \mathrm{a}$ & $5.4 a$ & $12.6 \mathrm{a}$ & $17.1 \mathrm{a}$ \\
\hline PBZ 500 & & 31 May & $53 \mathrm{bc}$ & $21 \mathrm{a}$ & $4.7 \mathrm{a}$ & $11.9 \mathrm{a}$ & $17.4 \mathrm{a}$ \\
\hline PBZ 500 & & 31 May, 4 June & $59 \mathrm{ab}$ & $19 \mathrm{a}$ & $4.4 \mathrm{a}$ & $11.3 \mathrm{a}$ & $16.4 \mathrm{a}$ \\
\hline UCZ 250 & & 31 May & $53 \mathrm{bc}$ & $28 \mathrm{a}$ & $5.0 \mathrm{a}$ & $11.7 \mathrm{a}$ & $16.7 \mathrm{a}$ \\
\hline UCZ 250 & & 31 May, 4 June & $56 \mathrm{abc}$ & $24 \mathrm{a}$ & $5.0 \mathrm{a}$ & $12.6 \mathrm{a}$ & $17.1 \mathrm{a}$ \\
\hline \multicolumn{8}{|l|}{ Stion ${ }^{y}$} \\
\hline Geneva/N & .106 & & $53 \mathrm{~b}$ & $21 \mathrm{a}$ & $4.8 \mathrm{a}$ & $13.0 \mathrm{a}$ & $18.7 \mathrm{a}$ \\
\hline Macspur/l & 1.106 & & $53 \mathrm{~b}$ & $27 \mathrm{a}$ & $5.0 \mathrm{a}$ & $11.9 \mathrm{ab}$ & $16.5 \mathrm{~b}$ \\
\hline Morspur/l & 4.106 & & $62 \mathrm{a}$ & $19 \mathrm{a}$ & $5.2 \mathrm{a}$ & $12.4 \mathrm{ab}$ & $16.6 \mathrm{~b}$ \\
\hline Macspur/ & h.111 & & $54 \_b$ & $18 \mathrm{a}$ & $4.9 \mathrm{a}$ & $11.5 \mathrm{~b}$ & $16.5 \mathrm{~b}$ \\
\hline
\end{tabular}

${ }^{\mathrm{z}}$ Applications with an air-blast sprayer at 1700 (31 May), 2000 (4 June), or 2250 liters $\cdot \mathrm{ha}^{-1}$ (28 June). Full bloom 17 May.

yean separation within factors and columns by Duncan's multiple range test $(P=0.05)$. No chemical $\times$ stion interactions. Each mean for chemical contains eight observations, for stion 12 observations.

in fruit treated with the double application of PBZ and either UCZ treatment compared to the control. 'Morspur' produced smaller fruit, 'Macspur' lower flesh firmness, and 'Macspur'/ MM.106 lower SSC than the nonspur 'Geneva McIntosh' in 1988.

Following 19 weeks of air storage, flesh firmness after 1 day or 1 week at room temperature was similar for all growth regulator treatments (Table 8). Differences in SSC were similar to those at harvest. Ethylene evolution was reduced for fruit treated with DZ or once with UCZ, but disorders were unaffected by any growth regulator. Stion differences in flesh firmness and SSC were similar to those at harvest, but ethylene evolution and incidence of disorders following storage were not.

\section{Discussion}

Unlike DZ, application of PBZ later than 5 WAFB had little influence on 'McIntosh' apples at harvest or after storage, although incidence of stem-cavity browning and brown core was increased by the later PBZ treatments in 1987, the season in which these applications contained a surfactant. PBZ applications within 5 WAFB increased skin red color and reduced flesh firmness loss and preharvest drop, but these effects were not consistent from year to year. Luo et al. (1989) found a similar limit on PBZ application time in relation to flesh firmness loss. By 5 weeks after petal fall, PBZ application had produced no effect on tissue firmness at harvest in 'Golden Delicious' and following storage in both 'Bramley's Seedling' and 'Golden Delicious'. 
Table 7. Effects of daminozide (DZ), paclobutrazol (PBZ), and uniconazole (UCZ) foliar applications in 1988 on 'McIntosh' fruit quality characteristics at harvest in 1988 (Expt. 3).

\begin{tabular}{|c|c|c|c|c|c|c|}
\hline Factor & $\begin{array}{c}\text { Application } \\
\text { date(s) }\end{array}$ & $\begin{array}{c}\text { Fruit } \\
\text { wt } \\
(\mathrm{g}) \\
\end{array}$ & $\begin{array}{l}\text { Flesh } \\
\text { firmness } \\
(\mathrm{N})\end{array}$ & $\begin{array}{c}\text { Soluble } \\
\text { solids } \\
\text { concn } \\
(\%)\end{array}$ & $\begin{array}{l}\text { Starch } \\
\text { index }\end{array}$ & $\begin{array}{c}\mathrm{C}_{2} \mathrm{H}_{4} \\
\left(\mu \mathrm{l} \cdot \mathrm{kg}^{-1} \cdot \mathrm{hr}^{-1}\right)\end{array}$ \\
\hline \multicolumn{7}{|c|}{ Chemical (mg-liter $\left.{ }^{-1}\right)^{y, x}$} \\
\hline Control & -- & $140 \mathrm{a}$ & $63.4 \mathrm{~b}$ & $12.9 \mathrm{a}$ & $3.5 \mathrm{ab}$ & $5.9 \mathrm{ab}$ \\
\hline DZ 750 & 28 June & $140 \mathrm{a}$ & $66.1 \mathrm{a}$ & $12.9 \mathrm{a}$ & $3.2 \mathrm{~b}$ & $0.4 \mathrm{~b}$ \\
\hline PBZ 500 & 31 May & $140 \mathrm{a}$ & $65.0 \mathrm{ab}$ & $12.7 \mathrm{ab}$ & $3.4 \mathrm{ab}$ & $3.2 \mathrm{ab}$ \\
\hline PBZ 500 & 31 May, 4 June & $140 \mathrm{a}$ & $65.6 \mathrm{a}$ & $12.5 \mathrm{~b}$ & $3.6 \mathrm{a}$ & $4.3 \mathrm{ab}$ \\
\hline UCZ 250 & 31 May & $140 \mathrm{a}^{\circ}$ & $65.1 \mathrm{ab}$ & $12.4 \mathrm{~b}$ & $3.4 \mathrm{ab}$ & $9.9 \mathrm{a}$ \\
\hline UCZ 250 & 31 May, 4 June & $150 \mathrm{a}$ & $64.9 \mathrm{ab}$ & $12.5 \mathrm{~b}$ & $3.6 \mathrm{a}$ & $7.8 \mathrm{a}$ \\
\hline \multicolumn{7}{|l|}{ Stion $^{x}$} \\
\hline Geneva/MM.106 & & $140 \mathrm{ab}$ & $66.4 \mathrm{a}$ & $12.6 \mathrm{a}$ & $3.4 \mathrm{a}$ & $3.2 \mathrm{a}$ \\
\hline Macspur/MM.106 & & $150 \mathrm{a}$ & $64.7 \mathrm{~b}$ & $12.4 \mathrm{~b}$ & $3.4 \mathrm{a}$ & $8.2 \mathrm{a}$ \\
\hline Morspur/MM.106 & & $130 \mathrm{~b}$ & $65.5 \mathrm{ab}$ & $12.7 \mathrm{a}$ & $3.6 \mathrm{a}$ & $5.5 \mathrm{a}$ \\
\hline Macspur/MM.111 & & $140 \mathrm{ab}$ & $63.1 \mathrm{c}$ & $12.8 \mathrm{a}$ & $3.3 \mathrm{a}$ & $4.1 \mathrm{a}$ \\
\hline \multicolumn{7}{|l|}{ Harvest date } \\
\hline 6 Sept. & & $130 \mathrm{~b}$ & $66.9 \mathrm{a}$ & $12.5 \mathrm{~b}$ & $3.0 \mathrm{~b}$ & $3.4 \mathrm{~b}$ \\
\hline 13 Sept. & & $150 \mathrm{a}$ & $63.0 \mathrm{~b}$ & $12.8 \mathrm{a}$ & $3.9 \mathrm{a}$ & $7.1 \mathrm{a}$ \\
\hline
\end{tabular}

${ }^{2}$ Mean treatment rating on a scale from 1 to 9 (immature to overmature).

${ }^{y}$ Applications with an air-blast sprayer at 1700 (31 May), 2000 (4 June), or 2250 liters $\cdot$ ha $^{-1}$ (28 June). Full bloom 17 May.

${ }^{x}$ Mean separation within factors and columns by Duncan's multiple range test ( $P=0.05$ ) for- chemical and stion and by $\mathrm{F}$ test $(P=0.05)$ for harvest date. No interactions among factors. Each mean for chemical contains 16 observations, for stion 24 observations, and for harvest date 48 observations.

Table 8. Effects of daminozide (DZ), paclobutrazol (PBZ), and uniconazole (UCZ) foliar applications in 1988 on 'McIntosh' fruit quality characteristics following 19 weeks of storage in air at $0.5 \mathrm{C}$ (Expt. 3).

\begin{tabular}{|c|c|c|c|c|c|c|c|c|}
\hline \multirow[b]{2}{*}{ Factor } & \multirow{2}{*}{$\begin{array}{l}\text { Application } \\
\text { date(s) }\end{array}$} & \multicolumn{2}{|c|}{$\begin{array}{c}\text { Flesh } \\
\text { firmness } \\
(\mathrm{N})\end{array}$} & \multirow{2}{*}{$\begin{array}{c}\text { Soluble } \\
\text { solids } \\
\text { concn } \\
(\%)\end{array}$} & \multirow{2}{*}{$\begin{array}{c}\mathrm{C}_{2} \mathrm{H}_{4} \\
\left(\mu \mathrm{l} \cdot \mathrm{kg}^{-1} \cdot \mathrm{hr}^{-1}\right)\end{array}$} & \multirow{2}{*}{$\begin{array}{c}\text { Stem } \\
\text { cavity } \\
\text { browning } \\
(\%)^{z}\end{array}$} & \multirow{2}{*}{$\begin{array}{c}\text { Brown } \\
\text { core } \\
(\%)^{z} \\
\end{array}$} & \multirow{2}{*}{$\begin{array}{c}\begin{array}{c}\text { Internal } \\
\text { breakdown } \\
(\%)^{z}\end{array} \\
\end{array}$} \\
\hline & & 1 day & 1 week $^{z}$ & & & & & \\
\hline \multicolumn{9}{|c|}{ Chemical (mg $\cdot$ liter $\left.^{-1}\right)^{y, x}$} \\
\hline Control & --- & $40.7 \mathrm{a}$ & $38.8 \mathrm{a}$ & $12.9 \mathrm{ab}$ & $158 \mathrm{a}$ & $28 \mathrm{a}$ & 45 a & 13 a \\
\hline $\mathrm{DZ} 750$ & 28 June & $41.4 \mathrm{a}$ & $39.9 \mathrm{a}$ & $13.0 \mathrm{ab}$ & $137 \mathrm{c}$ & $27 \mathrm{a}$ & $42 \mathrm{a}$ & $14 a$ \\
\hline PBZ 500 & 31 May & $41.4 \mathrm{a}$ & $39.4 \mathrm{a}$ & $12.7 \mathrm{abc}$ & $152 a b$ & 31 a & $30 \mathrm{a}$ & $9 \mathrm{a}$ \\
\hline PBZ 500 & 31 May, 4 June & $41.7 \mathrm{a}$ & $39.8 \mathrm{a}$ & $12.6 \mathrm{bc}$ & $151 \mathrm{ab}$ & 32 a & $37 \mathrm{a}$ & $12 \mathrm{a}$ \\
\hline UCZ 250 & 31 May & $41.1 \mathrm{a}$ & $39.6 a$ & $12.4 \mathrm{c}$ & $144 \mathrm{bc}$ & $45 \mathrm{a}$ & $40 \mathrm{a}$ & 8 a \\
\hline UCZ 250 & 31 May, 4 June & $41.1 \mathrm{a}$ & $39.2 \mathrm{a}$ & $12.4 \mathrm{c}$ & $161 \mathrm{a}$ & $38 \mathrm{a}$ & $42 \mathrm{a}$ & $13 \mathrm{a}$ \\
\hline \multicolumn{9}{|l|}{ Stion $^{\mathbf{x}}$} \\
\hline Geneva/MM.106 & & $42.4 \mathrm{a}$ & $40.7 \mathrm{a}$ & $12.8 \mathrm{a}$ & $151 \mathrm{ab}$ & $22 b$ & $43 a$ & $7 \mathrm{c}$ \\
\hline Macspur/MM.106 & & $40.3 \mathrm{~b}$ & $39.1 \mathrm{~b}$ & $12.4 \mathrm{~b}$ & $143 \mathrm{~b}$ & $46 \mathrm{a}$ & $45 \mathrm{a}$ & $14 \mathrm{~b}$ \\
\hline Morspur/MM.106 & & $42.4 \mathrm{a}$ & $40.2 \mathrm{a}$ & $12.6 \mathrm{ab}$ & 158 a & 34 a & 34 a & $5 \mathrm{c}$ \\
\hline Macspur/MM.111 & & $39.7 \mathrm{~b}$ & $37.9 \mathrm{c}$ & $12.9 \mathrm{a}$ & $151 \mathrm{ab}$ & $34 a$ & 35 a & $23 a$ \\
\hline \multicolumn{9}{|l|}{ Harvest date ${ }^{x}$} \\
\hline $6 \mathrm{Sept}$ & & $42.0 \mathrm{a}$ & $40.3 \mathrm{a}$ & $12.4 \mathrm{~b}$ & 152 a & 34 a & $34 \mathrm{~b}$ & $10 \mathrm{a}$ \\
\hline 13 Sept. & & $40.5 \mathrm{~b}$ & $38.6 \mathrm{~b}$ & $12.9 \mathrm{a}$ & 149 a & $34 \mathrm{a}$ & $45 \mathrm{a}$ & $13 \mathrm{a}$ \\
\hline
\end{tabular}

${ }^{2}$ Evaluation after 1 week at room temperature. Percentages are of 25 -fruit samples showing moderate (rating of 2) to severe (rating of 3) symptoms.

${ }^{y}$ Applications with an air-blast sprayer at 1700 (31 May), 2000 (4 June), or 2250 liters $\cdot$ ha $^{-1}$ (28 June). Full bloom 17 May.

${ }^{x}$ Mean separation within factors and columns by Duncan's multiple range test $(P=0.05)$ for chemical and stion and by $\mathrm{F}$ test $(P$ $=0.05)$ for harvest date. No interactions among factors. Each mean for chemical contains 16 observations, for stion 24 observations, and for 'harvest date 48 observations.

Luo et al. (1987) found the same effective PBZ applicationtime range for inducing a delay in the respiratory climacteric immediately after harvest and storage, but the differences in respiration required $\approx 19$ days from harvest or 5 days from storage to appear and the delay was only $\approx 3$ days long. Respiration data in the trials reported here were only recorded for a maximum of 3 days and showed little effect of DZ, PBZ, or UCZ (data not shown). Ethylene evolution at harvest was lower for the DZ treatment in 1987 than for untreated fruit and after storage for DZ in 1987 and 1988. No PBZ treatment affected ethylene production at harvest in 1987 or 1988, and ethylene evolution after storage was lower only for the later PBZ treatments in 1987 and the UCZ treatments in 1988. Interpretation of these ethylene evolution levels is complicated by the absence 
of sequential sampling through time, which would establish the pattern of the change in ethylene evolution. Luo et al. (1987) found a similar responsive period after flowering for reduction of ethylene evolution by PBZ as for respiration delay, but no clear evidence for a similar PBZ application-time effect on respiration was found in the trials reported here.

Luo et al. (1989) also reported reductions in fruit SSC from PBZ treatments at first bloom or petal fall only. Reduced SSC was observed only in 1988 in trials reported here and only in treatments receiving UCZ or the double application of PBZ.

Foliar mineral-nutrient analyses showed no differences in macronutrient levels in any of the three trials reported here (data not shown). Luo et al. (1989) found increased $\mathrm{Ca}$ and $\mathrm{P}$ and decreased $\mathrm{K}$ in fruit treated with PBZ at first bloom or petal fall. We do not know whether such changes might explain the retardation in flesh-firmness loss observed for some of the PBZ treatments in 1987 and 1988 in trials reported here. Luo et al. (1987, 1989) did not report foliar mineral-nutrient levels.

Stions differed in flesh firmness, SSC, and in some indices of fruit deterioration in our trials. Stion did not influence the starch index or ethylene evolution. Stion differences were similar in magnitude, but not necessarily in direction, to those reported in earlier studies (Lane and Meheriuk, 1976; Meheriuk and Lane, 1983). Stion differences observed here were likely of little economic significance (Lane and Meheriuk, 1976).

PBZ treatments reducing flesh firmness loss and preharvest drop did not produce carryover effects on fruit pedicel length, a sensitive indicator of carryover in 'McIntosh' (Elfving and Proctor, 1986). UCZ proved ineffective for producing beneficial changes in fruit characteristics in one trial. Neither PBZ nor UCZ produced the range or consistency of effects of DZ nor showed the capacity for controlling 'McIntosh' fruit maturation and ripening characteristic of DZ (Edgerton and Blanpied, 1970; Greene et al., 1977; Looney, 1968a, 1968b, 1971, 1975a, 1975b; Miller and Lougheed, 1971).

In their study, Luo et al. (1987) pointed out that the delay in fruit-ripening ascribed to PBZ was small. However, Luo et al. (1989) concluded that despite reduced SSC, PBZ-mediated improvement in flesh firmness -and mineral-nutrient content "offers an important advantage to the commercial fruit grower". The changes in fruit response reported for PBZ and UCZ in our studies, although measurable, were not of practical significance, and higher quantities of PBZ are unlikely to be useful because of residual effects (Elfving and Proctor, 1986). The studies reported here do not support the concept that PBZ or UCZ at moderate application levels can be used effectively to replace DZ on 'McIntosh' apple.

\section{Literature Cited}

Agriculture Canada. 1987. Canada Agricultural Products Standards Act. Dairy, Fruit and Vegetable Division, Agr. Canada, Ottawa, Ont., Canada.

Bodnar, J. M., J.T.A. Proctor, J.E. Laing, and R.A. Cline. 1983. Nutrient changes in apple leaves due to the spotted tentiform leafminer. J. Amer. Soc. Hort. Sci. 108:681-685.

Edgerton, L.J. and G.D. Blanpied. 1970. Interaction of succinic acid 2,2-dimethyl hydrazide, 2-chloroethylphosphonic acid and auxins on maturity, quality and abscission of apples. J. Amer. Soc. Hort. Sci. 95:664-666
Elfving, D.C. and J.T.A. Proctor. 1986. Long-term effects of paclobutrazol (Cultar) on apple-tree shoot growth, cropping and fruit-leaf relations. Acts Hort. 179:473-480.

Elfving, D. C., C.L. Chu, E.C. Lougheed, and R.A. Cline. 1987. Effects of daminozide and paclobutrazol treatments on fruit ripening and storage behavior of 'McIntosh' apple. J. Amer. Soc. Hort. Sci. 112:910-915.

Forshey, C.G. 1970. The use of Alar on vigorous 'McIntosh' apple trees. J. Amer. Soc. Hort. Sci. 95:64-67.

Forshey, C.G. and D.C. Elfving. 1979. Branch samples for yield and fruit size comparisons in apple. HortScience 14:143-144.

Greene, D.W., W.J. Lord, and W.J. Bramlage. 1977. Mid-summer applications of ethephon and daminozide on apples. I. Effect on 'McIntosh'. J. Amer. Soc. Hort. Sci. 102:491-494.

Lane, W.D. and M.M. Meheriuk. 1976. McIntosh apples compared with fruit of three spur strains. Can. J. Plant Sci. 56:847-851.

Looney, N.E 1968a. Inhibition of apple ripening by succinic acid 2,2dimethylhydrazide and its reversal by ethylene. Plant Physiol. 43:11331137.

Looney, N,E. 1968b. Control of apple ripening by succinic acid 2,2dimethylhydrazide, 2-chloroethyltrimethylammonium chloride, and ethylene. Plant Physiol. 44: 1127-1131.

Looney, N.E. 1971. Interaction of ethylene, auxin, and succinic acid2,2-dimethylhydrazide in apple fruit ripening control. J. Amer. Soc. Hort. Sci. 96:350-353.

Looney, N.E. 1975a. Control of ripening in 'McIntosh' apples. I. Some growth regulator effects on preharvest drop and fruit quality at four harvest dates. J. Amer. Soc. Hort. Sci. 100:330-332.

Looney, N.E. 1975b. Control of ripening in 'McIntosh' apples. H. Effect of growth regulators and $\mathrm{CO}$, on fruit ripening, storage behavior and shelf life. J. Amer. Soc. Hort. Sci. 100:332-336.

Lougheed, E.C. and E.W. Franklin. 1968. Automated system for measuring respiration. Can. J. Plant Sci. 48:435-438.

Lougheed, E. C., E.W. Franklin, and R.B. Smith. 1969. Ethylene analyses by automatic gas chromatography. Can. J. Plant Sci. 49:386391.

Luo, Y., H. Wainwright, and K.G. Moore. 1987. Effects of orchard application of paclobutrazol on the post-harvest ripening of apples. J. Hort. Sci. 62:295-301.

Luo, Y., H. Wainwright, and K.G. Moore. 1989. Effects of orchard applications of paclobutrazol on the composition and firmness of apple fruits. Scientia Hort. 39:301-309.

Meheriuk, M.M. and W.D. Lane. 1983. A comparison of fruit from spur and standard 'McIntosh' at harvest and after controlled-atmosphere storage. HortScience 18:220-221.

Miller, S.R. and E.C. Lougheed. 1971. Residual effects of Alar on the carbon dioxide and ethylene production of McIntosh apples at harvest and after long storage. Can. J. Plant Sci. 51:87-91.

Phillips, W.R. and P.A. Poapst. 1952. Storage of apples. Can. Dept. of Agr., Hort. Div., Expt. Farms Serv. Publ. 776.

Poapst, P. A., G.M. Ward, and W.R. Phillips. 1959. Maturation of McIntosh apples in relation to starch loss and abscission. Can. J. Plant Sci. 39:257-263.

Priest, K.L. and E.C. Lougheed. 1981. Evaluating apple maturity using the starch-iodine test. Ontario Min. Agr. and Food Factsheet 81025 .

Prive, J. P., D.C. Elfving, and J.T.A. Proctor. 1988. Pedicel characteristics of four apple cultivars. Fruit Var. J. 42:122-125.

Smith, R. B., E.C. Lougheed, E.W. Franklin, and I. McMillan. 1979. The starch iodine test for determining stage of maturation in apples. Can. J. Plant Sci. 59:725-735.

Williams, M. W., L.P. Batjer, and G.C. Martin. 1964. Effects of $N$ dimethyl amino succinamic acid (B-Nine) on apple quality. Proc. Amer. Soc. Hort. Sci. 85:17-19. 